\title{
Management of Bilateral Mandibular Fractures Using Intraoral Unilateral Application of Bone Plates in the Dromedary Camel
}

\author{
Gamal Karrouf $^{1,2^{*}}$,Awad Rizk ${ }^{2}$, El-Sayed El-Shafaey ${ }^{2,3}$, Khaled Abouelnasr ${ }^{2}$, Mohamed \\ Abou-Alsaud ${ }^{4}$, Mohamed Gomaa ${ }^{5}$, Ali Kandeel ${ }^{6}$ and Elie Barbour ${ }^{7}$ \\ ${ }^{1}$ Faculty of Science, Medical Physics Department, King Abdulaziz University, Jeddah 21589, \\ Saudi Arabia. \\ ${ }^{2}$ Department Surgery, Anesthesiology and Radiology Department, Faculty of Veterinary Medicine \\ Mansoura University, Mansoura, Egypt. \\ ${ }^{3}$ Department of Veterinary Medicine, College of Agriculture and Veterinary Medicine, \\ Qassim University, Buraydah 51452, PO Box 6622, Qassim, Saudi Arabia. \\ ${ }^{4}$ Faculty of Meteorology, Environment and Arid Land Agriculture, King Abdulaziz University, \\ Jeddah 21589, Saudi Arabia \\ 55 Department of Surgery, Anesthesiology and Radiology, Faculty of Veterinary Medicine, \\ Zagazig University, Zagazig, Egypt. \\ ${ }^{6}$ Dept.of biology, Fac. of Science and Arts, Jeddah University, Alkamil branch, Saudi Arabia. \\ ${ }^{7}$ Department of Animal and Veterinary Sciences, Faculty of Agricultural and Food Sciences, \\ American University of Beirut, Beirut, Lebanon; Adjunct Professor at the Biochemistry Department, \\ King Abdulaziz University, Jeddah, Kingdom of Saudi Arabia.
}

\begin{abstract}
A clinical study to evaluate unilateral application of 10 holes dynamic compression plates (DCPs) for the treatment of bilateral fracture of the mandible insingle-humped camels. Six cases of typical mandibular fractures in the dromedary camel were treated by unilateral application of $3.5 \mathrm{~mm} 10$ - holes DCP. Restrained of camels was performed with deep sedation using xylazine hydrochloride and ketamine hydrochloride. The main cause of mandibular fractures in the recorded cases was the biting. The result was quite encouraging and all the animals had an uneventful healing. No loosening of the fixation screws were noted in any case and all the fractures healed at variable time intervals ranging from 2.5 to 3 months period. We concluded that bilateral mandibular fracture in dromedary camels could be successfully repaired by unilateral bone plate with good outcome without additional fixation devices. At the present time, to our knowledge, this investigation could be considered the first report on the use unilateral intraoral DCP in clinical bilateral mandibular fractured cases of camels.
\end{abstract}

Keywords: Mandibular fractures, camel, intraoral, unilateral bone plate

\section{INTRODUCTION}

Mandibular fractures are common among camels in Saudi Arabia due to fighting with other male camels during rut season. The presence of mental canal and alveoli of tusks makes this area more weak and susceptible to fractures (1-3).

Different surgical techniques for repairs have been used for the fixation of mandibular fracture with variable results such as transfixion pins along with plaster of Paris bandaging $(1,3)$; wiring between incisors and 1st premolars(2); bone plating(4); plaster of Paris bandage and a wooden plate as a splint (5); interdental wiring (6) and interdental wiring or application of a Ushaped aluminum bar (7-9).

Methods of internal fixation and fracture repair in small animals are a commonplace, but these methods still have some drawbacks in the large animals. Different means of external and internal fixation of fractures have been described in llamas (10) ;llamas and small ruminants(11);camelids (12); alpacas (13).A comprehensive discussion on complications and restrictions after fracture repair in alpacas has been documented (14). 
Aim: In this paper, we report cases of bilateral fracture of the mandible in a dromedary camel that was successfully repaired using unilateral intraoral bone plating using DCP.

\section{MATERIALS AND MethodS}

\subsection{Animals}

six male breeding camels (Camelus dromedarius) between 8-12 years of age weighing about 400-600 $\mathrm{kg}$ suffering from mandibular bilateral fracture (Fig.1) were repaired by intraoral unilateral bone plating under deep sedation of the camel by xylazineHCl $(0.3 \mathrm{mg} / \mathrm{kg}$ body weight(xylaject ADWIA, Egypt) and ketamine HCL $(0.3 \mathrm{mg} / \mathrm{kg}$ body weight given intravenously.

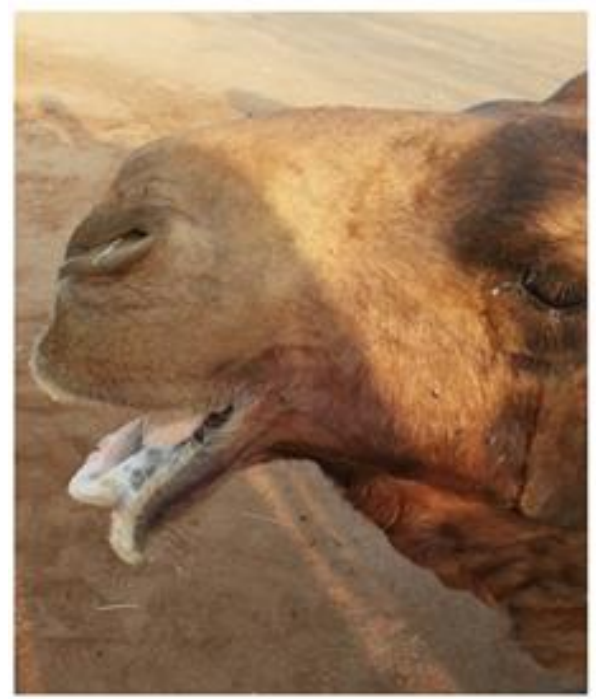

Figure 1. Bilateral mandibular fracture in a camel.

\subsection{Operative Procedure}

The operative site was prepared for aseptic surgery .The fracture site is exposed through intraoral incision. The blood clots and tissue debris if any are removed carefully and the fracture fragments are reduced and held in position. A $3.5 \mathrm{~mm} \mathrm{10-hole} \mathrm{DCP} \mathrm{was} \mathrm{contoured} \mathrm{according} \mathrm{to} \mathrm{the} \mathrm{bone} \mathrm{curvature}$ slightly over-bent. $2,7 \mathrm{~mm}$ holes are drilled through the bone approximately $1 \mathrm{~cm}$ from fractured line and, tapped with $3.5 \mathrm{~mm}$ bone tap, measured with the plate in place and a 3.5 -mm cortical screw of proper length are inserted until it begins to engage the plate holes. The fracture is reduced and held in position with the bone-holding forceps. After tightening both of these first screws alternately the fracture gap is closed and the fragments reduced with good stability, The bone clampsare then removed, and the remaining holes on both sides of the fracture site were drilled ,measured, tapped, and the screws of proper length are inserted (Fig.2).After fracture fixation, an oral mucosal wound were cleaned and was opposed with continuous suture line using USP-1 polyglactin 910 acid suture material (Ethicon Coated Vicryl, Johnson \& Johnson International).

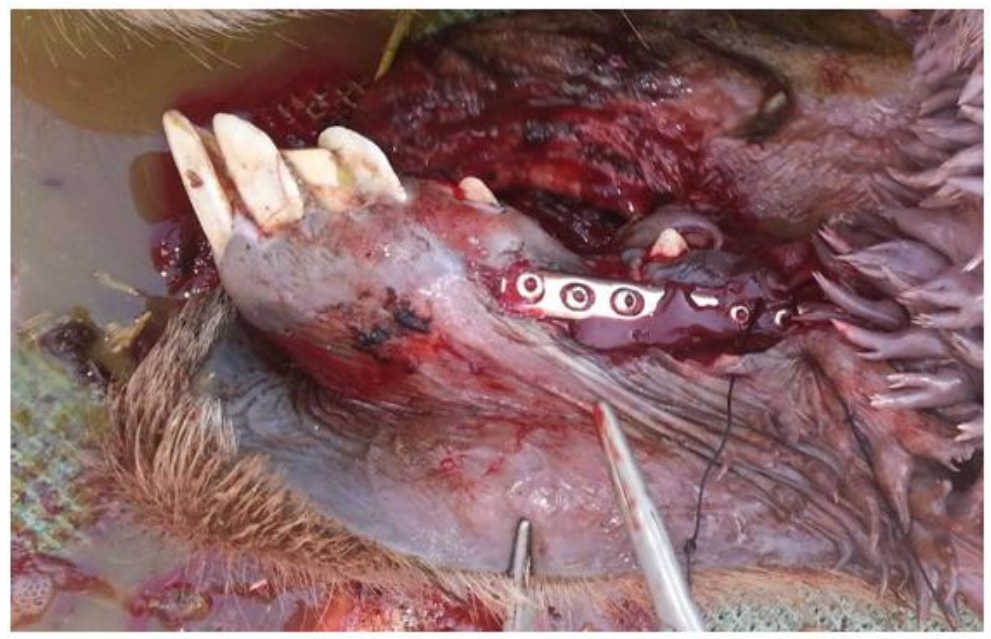

Figure 2. Intraoral incision and application of dynamic compression plate. 


\subsection{Postoperative Care}

The animals were administered an intramuscular injection of Ampidexlone (Coophavet, France) at the recommended dose rate of $1 \mathrm{ml} / 10 \mathrm{~kg}$ bodyweight daily for 10 days. Phenylbutazone $(5 \mathrm{mg} / \mathrm{kg}$ intravenously) was administered for 4 days. Camels had free access to water and allowed to feed, under supervision, only the semisolid food for one week post-surgery, then green fodder and roughage were given by hand to eat as The owner was advised to keep the animal confined alone in a separate capacious enclosure away from the other animals for a further period of eight weeks after which the camel was allowed free movement.

\section{RESULTS}

The main cause of mandibular fractures in the recorded cases was the biting in the present study; Fractures were treated by unilateral application of $3.5 \mathrm{~mm}$ 9- holes DCP. Analgesia obtained by xylazine and ketamine with the aforementioned dose was satisfactory enough for allowing plate applications. Axial compression was achieved through two $3.5 \mathrm{~mm}$ cortical screws placed eccentrically in cranio-caudal direction through the plate. 4 screws in the distal and four screws in the proximal fracture fragment were inserted. After that the fracture on one side of the bone is plated and the resultant axial compression was obtained, the fracture on the other side was sufficiently and perfectly reduced and stabilized (Fig.3).The healing occurred without complications where no deviations of the fracture fragment, no loosening of the fixation screws were noted in any case at variable time intervals in the postoperative period. The result was quite encouraging and all the animals had an uneventful healing and all the fractures healed at variable time intervals ranging from 2.5 to 3.5 months periods.

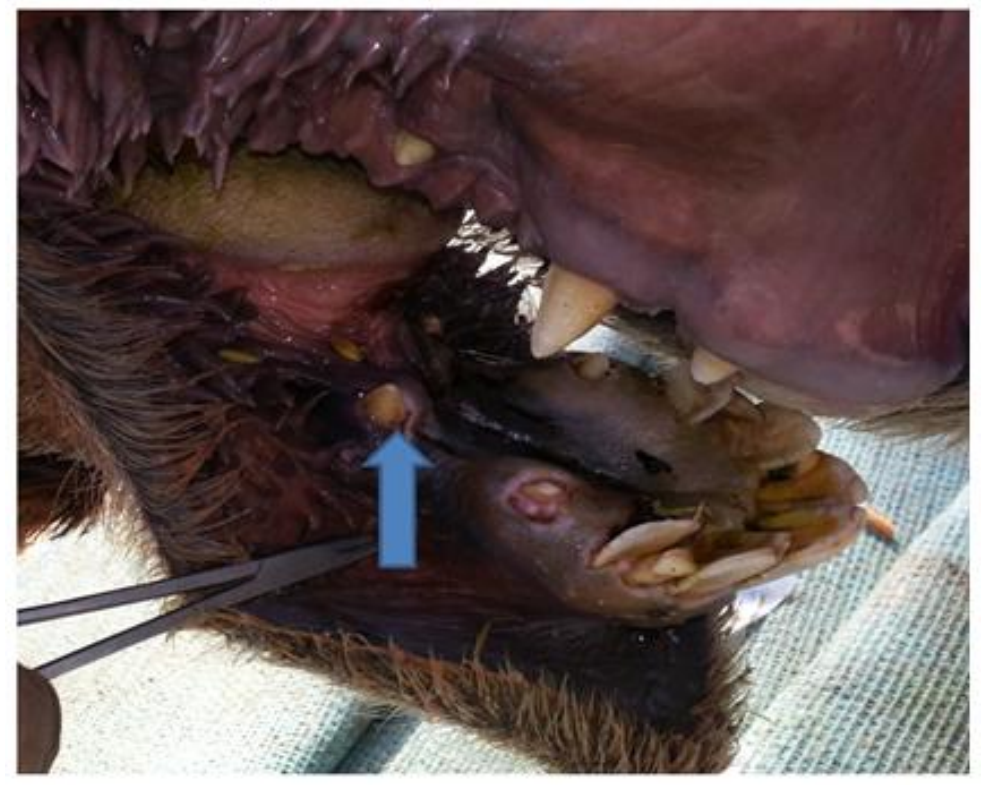

Figure 3. After application of DCP, the fracture on the other side was sufficiently and perfectly reduced and stabilized (arrow).

\section{DisCUSSION}

All cases of mandibular fractures were recorded in male camels and caused by camel biting during rutting season where sexually excited camel bulls often bite other camels. Trauma from another camel or handlers was not recorded as cause of mandibular fracture; these results are similar to previous studies $(\mathbf{1 5}, \mathbf{1 6})$. The rostral third of the horizontal ramus just rostral to the canines were involved. This is likely the weakest area of the mandible because of the presence of the rostral mental foramin, canine alveoli, and relatively small cross-sectional diameter (1). Also all cases of fracture recorded are opened one. This could be attribute to tightly applied oral mucosa is easily wounded resulting in an increase in frequency of open mandibular fractures $(6,8)$.

Axial compression resulted in good reduction of the fracture fragments and therefore, healing occurred without complications where the animal was able to move its mandible normally without any sort of deviation. A properly applied bone plate counteracts bending, rotational and shearing 
forces and appose fragment $(17,18)$. In our opinion, factors such as axial compression obtained by DCP, aseptic surgical procedure, provision of additional animal confinement for the first five postoperative weeks in a separate place with restricted activity played an important role towards good healing of the mandibular fractures (14).

The animal recovered from injury without major complications after 16 weeks of surgery, but was able to move its mandible normally without any sort of deviation $(12,13)$.It is recommended that fractures on both sides of the mandible should be plated or the fracture on the other side can be managed with interdental wiring technique (18). However, in our experience; if fracture on one side is perfectly stabilized, the fracture on the other side was sufficiently and perfectly reduced and stabilized so no other means of fixation was needed as recorded in the present cases.

The technique used here was nearly simple and practical for the fixation of bilateral fracture of the mandible with an objective of maintaining good immobilization of fractured ends during the healing period. The axial compression resulted in a perfect reduction of the fracture fragments and therefore, healing occurred without complications. Similar findings have been previously reported for repair of long bone fractures in South American camelids (12).

The DCP was used here as a self-compressing plate where the special geometry of its oval screws holes has increased the potential uses of the plate. The DCP does not require a tension device for axial compression and can be used to compress fractures without additional surgical exposure. Compression is achieved through eccentric placement of the screws in the oval holes of the plate. Because of the sloping design of the screw holes, the plate moves as the screw head is seated, screws can be put at an angle other than 90 degrees to the plate. Movement of the plate results in axial compression Also it should be slightly pre-stressed or over-bent. Pre-stressing a plate results in a 1 to 2- mm gap at the near cortex located under the plate but result in compression on the far cortex, thus increasing overall stability (19).

\section{Conclusion}

Bilateral Mandibular fractures in the present study were caused by bites and were successfully repaired by intraoral application of unilateral DCP with good outcome.

\section{REFERENCES}

[1] Bhatia YS, Misra SS, Lavania JP, et al. Surgical management of bilateral mandibular fracture in camel. Indian Vet J 1978; 2:85-87.

[2] Manfield GW, Tinson AH. Camels A compendium.University of Sydney Post Graduate Foundation in Veterinary Science.The TG Hungerford Vade Mecum Series for Domestic Animals, Series C; No. 22, 1996.

[3] Gahlot TK, Chouhan DS: Fractures in dromedary (Camelusdromedarius) - A retrospective study. J Camel PractRes 1994; 1:9-14.

[4] Kumar R, Singh G: Treatment of unilateral fracture of mandible by bone-plating in camels. Indian Vet J 1979; 56:58-59.

[5] Lavania JP. A field oriented immobilization technique for mandibular fracture in camel: A clinical study. Proceedings of the 3rd Annual Meeting for Animal Production under Arid Conditions 1998; 1: 174-179.

[6] Hanuman R, Gahlot TK, Ram H: Gross and radiological evaluation of RBR IDW technique for repair of mandibular fracture in camels (Camelusdromedarius). J Camel Pract Res 2001;8:199_ 202.

[7] Gahlot TK, Choudhary RJ, Chouhan DS, et al: Clinical evaluation of interdental wiring technique for mandibular fracture repair in camels. Indian Vet J 1989; 66:251-254.

[8] Ahmed F. Mandibular fracture in single-humped camels.Veterinary Surgery 2011 ;7: 903.

[9] Ahmed $F$ and Al-Sobayil F. Fractures in young, single-humped camels (Camelusdromedarius).Turk. J. Vet. Anim. Sci. 2012 36: 1-8.

[10] St. Jean, G., Bramlage, L.R., Constable, P.D.: Repair of fractureof the proximal portion of the radius and ulna in a llama. J. Am.Vet. Med. Assoc., 1989; 194: 1309-1311.

[11] Ramadan, R.O. and Al-Mubarak, A.I. . Fracture of proximal part of Radius and Ulna in camels. Proc. Int. Camel Conf. Bekaner, India; 2007,16-17 : 176-177. 
[12] Tee, S.Y., Dowling, B.A. and Dart, A.J. 2005. Treatment of long bone fractures in South American camelids: 5 cases. Aust. Vet. J. 83: 418-420.

[13] Newman, K.D., Anderson, D.E.: Humerus fractures in llamas and alpacas: seven cases (19982004). Vet. Surg. 2007; 36: 68-73.

[14] Semevolos, S.A., Huber, M.J., Parker, J.E., Reed, S.K.: Complications after orthopedic surgery in alpacas and llamas: 24 cases (2000-2006). Vet. Surg., 2008; 37: 22-26.

[15] Ramadan, R.O.: Fractures. In: Ramadan, R.O. Ed. Surgery and Radiology of the Dromedary Camel. 1st ed. King Faisal University, Kingdom of Saudi Arabia, 1994; 226-250.

[16] Gahlot TK: Fractures, in Gahlot TK (ed): Selected Topics on Camelids. Sugan Niwas ChanfanSagar Well, Bikaner, India, Sankhla Printers, 2000, pp 382-407.

[17] Smith BA, Kerwin SC, Hosgood G, Voyiadjis G, Echle R and Strain, GM: Mechanical comparison of two methods for Interfragmentary fixation in a short oblique fracture model. Vet. Comp. Orthop. Traumatol 1996 9:145-148.

[18] Siddiqui, MI and Telfah, MN. Interfragmental compression with $4.5 \mathrm{~mm}$ cortical screw. In: A Guide Book of Camel Surgery. 1st Ed. Abu Dhabi Food Control Authority, United Arab Emirates2010; pp: 135.

[19] Nunamaker DM, Perren SM: A radiological and histological analysis of fracture healing using prebending of compression plates. Clin Orthop 1979; 138: 167. 